\title{
REMARKS ON THE PROGRESS OF CELESTIAL MECHANICS SINCE THE MIDDLE OF THE CENTURY.
}

\section{PRESIDENTIAL ADDRESS DELIVERED BEFORE THE AMERICAN MATHEMATICAL SOCIETY, DECEMBER 27, 1895.}

BY DR. G. W. HILL.

The application of mathematics to the solution of the problems presented by the motion of the heavenly bodies has had a larger degree of success than the same application in the case of the other departments of physics. This is probably due to two causes. The principal objects to be treated in the former case are visible every clear night, consequently the questions connected with them received earlier attention; while, in the latter case, the phenomena to be discussed must ofttimes be produced by artificial means in the laboratory; and the discovery of certain classes of them, as, for instance, the property of magnetism, may justly be attributed to accident. A second cause is undoubtedly to be found in the fact that the application of quantitative reasoning to what is usually denominated as physics generally leads to a more difficult department of mathematics than in the case of the motion of the heavenly bodies. In the latter we have but one independent variable, the time; while in the former generally several are present, which makes the difference of having to integrate ordinary differential equations or those which are partial. Thus it happens that, while the science of astro-mechanics is started by Newton, that of thermal conductivity receives its first treatment, at the hands of Fourier, more than a century later. In addition to these two causes, ever since the discovery of the telescope the application of optical means to the discovery of whatever might be found in the heavens has always had a fascination for mankind. And, as the ability to co-ordinate and correlate the facts observed much enhances the enjoyment of scientific occupation, it has resulted that many who began as observers ended as mathematical astronomers. Thus our science has had relatively a large number of cultivators.

A thoroughly satisfactory history of our subject is yet to be written. We have only either slight sketches of the whole, or elaborate treatments of special divisions of the science, and none of them coming down to recent times. Among the former may be mentioned Gautier's Essai historique sur le pro- 
bleme des trois corps, which appeared in 1817. Also Laplace's historical chapters in the last volume of the Mécanique Céleste. Todhunter's History of the theories of attraction and the figure of the earth is an example of the latter class. Such books as Todhunter's - of which Delambre has given an earlier example in his Histoire de l'Astronomie - can hardly be regarded as history; they resemble rather extensive tables of contents of the literature examined, accompanied by short comments. However, in many cases, they are more useful to the student than formal histories would be, as, when judiciously compiled, they may, as epitomes in our libraries, take the place of a large mass of scientific literature. The History of Physical Astronomy, by Robert Grant, is a book that comes down to 1850, and professedly covers the whole of our subject. But only one third of this book is devoted to astro-mechanics, the rest dealing with what is really observational and descriptive astronomy. Moreover, the author indulges so much in diffusive veins of writing, that but a small fraction of the 200 pages is really given to purely historic statement. As far as the Lunar Theory is concerned, the third volume of M. Tisserand's Traite de Mécanique Céleste constitutes a fair history. But it must be borne in mind that the author's plan is to notice only the disquisitions having a first-class importance; hence his history is incomplete in this respect.

In America we are not well situated for investigations of this character; on account of the meagreness of our libraries. Of no inconsiderable number of memoirs and even books, having at least some importance in our subject, there exist no copies in the United States. Hence, should an American be inclined to undertake the task of writing the history of our subject, he must at least perform some of the work abroad.

In the present discourse it is proposed to touch very lightly the more important steps made since the middle of the century, the time at our disposal not admitting fuller treatment.

And first we will take up Delaunay's method, proposed for employment in the lunar theory, but quite readily extended to all classes of problems in dynamics. The first sketch of this method, given of course by the author himself, appeared in the Comptes Rendus of the Paris Academy of Sciences, in 1846. It professes to be merely an extract from a memoir offered for publication in the collections of the Academy, which must, however, have been afterwards withdrawn to make place for the two volumes of the Théorie du Mouvement de la Lune. When this extract is compared with the earlier chapters of the latter work, it is perceived that Delaunay has, to some extent, modified and improved his method in the interim between 1846 and 1860. In this long period nothing appeared from the author on this subject. He must have been profoundly engaged in 
applying his method to the motion of the moon. Tisserand's exposition of this method is somewhat more brief than the author's own. But when the necessary modifications are introduced into Delaunay's procedures, to make them applicable to the more general case of the motion of a system of bodies, the establishment of the formulas can be rendered still more brief.

There is one point in reference to Delaunay's method which, as far as I am aware, has escaped notice. This method consists in a series of operations or transformations, in each of which the position of the moon in space is defined by six variables, the number three being doubled in order that the velocities, as well as the co-ordinates, may be expressed without differentials. The aim of the transformations is to make one half of these, which Poincaré has called the linear variables, continually approach constancy, while the other half, named the angular variables, continually approach a linear function of the time. But at any stage of the process the position of the moon, as well as its velocity, is definitely fixed by the six variables produced by the last transformation, provided that the proper degree of variability is attributed to them, just as, before any transformation was made, the six elements of elliptic motion, usually denominated osculating, defined them; the point of difference to be noticed being that the more the transformations are multiplied, the more complex becomes the character of the expression of the former quantities in terms of the latter. But, however great may be the number of transformations, the series evolved have always one consistent trait, viz., that the angular variables are involved in them only through cosines or sines of linear functions of these variables, the linear functions being formed with integral coefficients. Now, as in all this work we are obliged to employ infinite series, the question of their convergence is an extremely important one. The inquiry in this respect may be divided into two parts, mainly independent of each other. These are, convergence as respects the angular variables, and convergence as respects the linear variables. The first part is much the more simple. Regarding each of the coefficients of the series we employ as a whole, that is, representing it by a definite integral, it is quite easily perceived that the said series are both legitimate and convergent when, giving the angular variables the utmost range of values, still no two of the bodies can occupy the same point of space. In the contrary case the series are evidently divergent. 'This condition affords certain limiting conditions for the values of the linear variables. Could we trace these limiting conditions through all the transformations, and obtain by comparison the formulas to which these tend when the number of transformations is made infinite, we should be in possession of the conditions of stability of motion of the system of bodies. The second 
part of the inquiry relates to the expression of the mentioned coefficients by infinite series proceeding according to powers and products of certain parameters which are functions of the linear variables. It is well known that, in the case of elliptic elements, Laplace and Cauchy almost simultaneously showed that the series are convergent when the eccentricity does not exceed a fraction which is about two-thirds. The determination of the conditions of convergence, after certain transformations have been made in the signification of the elements, is undoubtedly a more complex problem; nevertheless, it seems to be within the competency of analysis as it exists at present.

The discovery of the criterion for the convergence of series proceeding according to powers and products of parameters is due to Cauchy, and is a most remarkable contribution to the science of mathematics. Supposing that the parameters begin from zero values, this criterion amounts to saying that the moment the function, which the series is to represent, ceases to be holomorphic, or becomes infinite, that moment the series ceases to be convergent. Consequently, if a space, having as many dimensions as there are parameters in the case, be conceived, and a surface be constructed in it formed by the consensus of all the points where the considered function ceases to be holomorphic, then, provided the values of the parameters define a point within this surface, that is, on the same side where lies the origin, the series will be convergent. Generally this surface will be closed, and, within it, the function will not take infinity as its value.

Without any mathematical reasoning the propriety of the principle just enunciated may be perceived. Since it is possible for the series in powers and products to give only one value for the function, the moment the latter may have any one of several values, the series fails to give them all; and, as there is no reason why any particular value should be selected, the conclusion must be that it does not represent any of them. Also, it is easy to see that, when the function takes infinity as its value, the series fails to represent it.

In applying this principle to the series involved in the treatment of the problem of many bodies by Delaunay's method, it appears, at first sight, as if we must have some finite representation of the coefficients in question in order to discover the particular points at which they cease to be holomorphic, such, for instance, as is given by an algebraic or transcendental equation. But this is not imperative, as it is often possible to make this discovery from certain recognized properties of the function considered, without being in possession of its form explicitly or implicitly. It appears probable that, in the class of cases considered, the mentioned coefficients can be represented by multiple definite integrals, all taken between the limits 0 
and $\pi$, the independent variables being those which have been denominated angular. Such functions are always holomorphic, provided that the expressions under the signs of integration are themselves holomorphic between the mentioned limits. If the statement just made be admitted, although it may be impossible to write explicitly the mentioned expressions, we may, nevertheless, be certain that they remain holomorphic, provided that the linear variables, which may be the same as the parameters considered, are so restricted in their range of values that no matter what values the angular variables receive, no distance between any two bodies of the system can vanish. Or, in other words, that the $R$ of Delaunay must never become infinite. Thus it seems probable that the conditions of convergence for Delaunay's series are precisely identical with those for the stability of motion of the system.

The series arising in Delaunay's method, as applied to the moon, contain five parameters; the number would be six were the moon's mass not neglected. We should also have six in the application of the method to two planets moving about the sun; however, should we employ the well-known functions $b_{s}{ }^{(i)}$ of Laplace, the number would be reduced to five. It ought to be possible, therefore, after the performance of a limited number of operations, to assign limiting values to these parameters, below which the series would certainly be convergent. This also involves the possibility of finding limits to the errors committed by truncating the series at a certain order of terms. Again, provided the time is limited to a certain interval, the capacity of these truncated series for representing the co-ordinates of the planets could be shown by giving superior limits to the errors necessarily involved.

One more remark may be made before we leave Delaunay's method. In every operation or transformation half the integrals are obtained without the intervention of the time, and from these solely are obtained the ranges of values for all the linear variables. As no integrating divisors appear in their expressions, it follows that the question of stability is not affected in any way by the vanishing of these. Moreover, the presence of a libration in the angle of operation does not necessitate any change in the procedure. The integrating divisors which appear in the expressions for the angular variables, obtained through quadratures, may cause difficulty, but this can generally be removed by a modification of the parameters employed in the development of the coefficients in series. Beyond this it does not seem necessary to attend particularly to the terms which Professor Gyldén has designated as critical.

To give a succinct idea of the scope of this method, it may be said that it is applicable whenever, in the system, the planets maintain their order of succession from the sun. In systems 
where that undergoes change, as is the case with the group of minor planets, supposing their action on each other is sensible, it is not applicable.

Delaunay's method has not yet received all the developments and applications it is susceptible of.

The treatise of Hansen on the shortest and most ready method of deriving the perturbations of the small planets was published in the interval 1857-1861. But as the principles on which it is founded had been elaborated and communicated to the public some years earlier, it is, perhaps, more properly to be assigned to the first half of the century. In consequence, I pass it over with this slight mention.

Perhaps the most conspicuous labors in our subject, during the period of time we consider, are those of Professor Gyldén and M. Poincaré. We will limit our attention, for the remainder of this discourse, to the consideration of these investigations.

Professor Gyldén began work with the methods of Hansen and was gradually led to modifications of them looking towards their use for indefinite lengths of time. This quality has latterly become imperative with him, and he has recently published the first volume of what is evidently intended to be a lengthy work entitled Traité Analytique des Orbites Absolues des Huit Planetes Principales. To show the drift of Professor Gyldén's investigations, we cannot do better than give an analysis of this volume. At the outset the author introduces a class of curves he names periphlegmatic, that is, curves which surround a flame. The definition of this sort of curve is that it describes continually the space between two concentric spheres, and, at every point, turns its concavity towards the intersection of the radius vector with the inner sphere. In an application to the solar system, the sun is supposed to occupy the common centre of the spheres. The investigation is at first limited to the case where this curve is plane. A differential equation of the second order is derived which the radius vector of this curve satisfies, the independent variable being the angle described. The perpendicular distance between the spheres is called the diastem. The spheres are supposed to be drawn so that they touch the curve at the points where the radius becomes a maximum or minimum. Thus, in some cases, the spheres are regarded as fixed, in others, as movable. In the latter case, however, the sum of their radii is supposed to remain constant. Thence we have two groups of periphlegmatic curves; those with constant and those with variable diastems. The author gives examples of both these groups, in most cases of which the line of apsides is variable, and considers the situation and density of the points of intersection of these curves with themselves. 
The idea of an absolute orbit of a planetary body is this : an oval symmetrical with regard to an axis movable in space. While the axis remains constant in length (the half of it is called the protometre), the velocity of its motion may vary, and the diastem may also vary. Professor Gyldén, however, admits into the expressions of these variations only terms whose period would become infinite did the planetary masses vanish. These terms he calls elementary. But elementary terms in the diastem and the longitude of the perihelion can produce terms in the co-ordinates having periods which differ but little from the time of revolution of the planet. These are also called elementary terms. But the two classes are distinguished, the first as being of the type (A), and the second as of the type (B). In all the formulas relative to this matter the author insists on keeping the arc described by the radius as the independent variable.

The co-ordinates are only approximately given by the preceding apparatus of expressions. They must then have certain complements added to them; these, however, are all composed of terms which would vanish with the planetary masses.

In deriving the elementary terms in the radius of a planet through the integration of a linear differential equation of the second order, Professor Gyldén attaches much price to his method of establishing the convergence of the series formed by the successive terms. As the latter are obtained through division by divisors of the order of the planetary masses, it might be feared that some of them would turn out to be very large. But the author prevents this by retaining in the coefficient of the dependent variable in the differential equation a quantity equivalent to the sum of the squares of all the coefficients in the integral. This is named the horistic or limiting function. It is plain such an expression could be introduced in the mentioned coefficient, provided that the linear equation is the truncated form of an equation containing the cube of the variable. And in the problem of planetary motion the approximations may always be so ordered that this shall be the case.

With regard to the co-ordinate which exhibits the departure of the planet from a fixed plane, Professor Gyldén does not greatly deviate from the procedure of Hansen in following the displacement of the instantaneous plane of the orbit. Only here, as in the preceding treatment of the radius, he would sharply distinguish the elementary and non-elementary terms.

At this point is introduced certain new nomenclature. As before we had diastem now we have anastem to denote the product of the radius and the sine of the inclination; and what has generally been called the true argument of the latitude is here called the anastematic argument. Any angular magnitudes which are constantly moving through the circumference are 
astronomic arguments; and when they have the same mean velocity of rotation they are isokinetic; and isokinetic arguments are homorhythmic when, in each revolution through the circumference, they always retake together the same corresponding points. In like manner, the true anomaly is the diastematic argument, and we have diastematic and anastematic coefficients and moduli. It will be seen from this that Professor Gyldén does not shrink from imposing on us the labor of learning new terms.

Thus far we have been engaged in deriving the equations of the path followed by a heavenly body; it remains to show how we may find the point on that path occupied by the body at a given moment. There is then necessary an equation between the time and the variable assumed as independent, that is, the orbit longitude, or, more properly, the amount of angle described by the radius vector. If we suppose the absolute orbit to be described by the planet so that equal areas are passed over by the radius in equal times, it is plain that, on the attainment of a given longitude, a definite amount of time must have elapsed since the epoch. This is what Professor Gyldén calls the reduced time; and he computes the difference between it and the actual time required by the theory of gravity for the planet to arrive at the stated direction. This mode of proceeding does not differ from Hansen's except in the point that the absolute orbit is substituted for a fixed ellipse.

But this gives us correctly only the orbit longitude; for the radius and the latitude, which correspond in the absolute orbit to this reduced time, are not quite those which the planet has at the actual time. Consequently, Professor Gyldén proposes to compute two corrections, the one to be applied to the product of the eccentricity into the cosine of the true anomaly, the other to the sine of the latitude. Also the reduction of the orbit longitude to the plane of reference must be manipulated so that it comes out correctly.

The employment of the orbit longitude as independent variable throughout all the integrations necessitates a mass of very intricate transformations of terms from one shape into another. Also the integrations which bear on elementary terms must be kept distinct from those which bear on non-elementary terms. A degree of complexity is thus imparted to the subject, which makes it difficult to see when one has really gathered up all the warp and woof of it. Professor Gyldén has nowhere removed the scaffolding from the front of his building and allowed us to see what architectural beauty it may possess; it is necessary to compare a large number of equations scattered through the volume before one can opine how the author means to proceed.

The advantages claimed for the method are that it prevents 
the time from appearing outside the trigonometrical functions, and that it escapes all criticism on the score of convergence. The first is readily conceded, but many simpler methods possessing this advantage are already elaborated, and it is not so clear that the second ought to be granted.

No completely worked out example of the application of this method has yet been published. The great labor involved will naturally deter investigators from employing it.

In 1890 was published the memoir of M. H. Poincaré, entitled Sur le problème des trois corps et les équations de la dynamique, and which obtained the prize of the King of Sweden. Most of the results of this memoir were worked over and presented anew with greater elaboration and clearness by their author in Les Méthodes Nouvelles de la Mécanique Céleste. Here we find a large number of new and very interesting theorems.

First is to be noted the class of particular solutions in the problem of the motion of a system of material points which are now named periodic solutions. The initial relative positions and velocities of the several points are so adjusted that, after the lapse of a definite time, the latter retake them. Hence is evident a method which may be employed to elaborate this special case of motion, viz., by the tentative process with mechanical quadratures. M. Poincaré has divided this sort of solutions into three classes, of which, however, the second and third are not essentially different. He has shown that, in the latter classes, the values of the arbitrary constants of the problem must be so adjusted that no secular inequalities, or, as Professor Gyldén calls them, elementary terms, may arise. The number and variety of these particular solutions is far greater than one would at first sight imagine.

We come now to a second class of particular solutions named by the author asymptotic. It arises from the consideration of solutions differing very little from periodic solutions. Here we have to deal with linear differential equations having periodic coefficients. The integrals of these contain in their terms exponential factors, and on the nature of the exponents of these factors depends the quality of the resulting solutions. M. Poincaré has named these exponents characteristic. They are roots of an algebraic equation of a degree equal to the number of dependent variables involved in the question. If any of these roots are imaginary with real portions or wholly real, we are in presence of asymptotic solutions. The algebraic equation mentioned contains the unknown only in even powers; hence the characteristic exponents are in pairs having the same absolute value, but with contrary signs. In all the cases presented by astronomy, where, on account of the near approach to circular motion, a periodic solution can be taken as 
a first approximation, it appears that the squares of the characteristic exponents are all real and negative. Thus, there is no call here to consider this sort of solution, and this fact must much diminish the interest of the astronomer in it. M. Poincaré has, however, elaborated it with great pains, showing how the effect of higher powers of the deviations from the periodic solution may be taken into account. The series resulting are, nevertheless, divergent, as in other cases.

The second volume of the Méthodes Nouvelles is devoted to the elaboration and consideration of various processes for developing the integrals of planetary motion according to the powers of a small parameter. The chief of these are due to Professor Newcomb and MM. Lindstedt and Bohlin; but M. Poincaré has augmented the number of them by introducing modifications of his own. All involve the principle of recurrence; that is, the first step is the only one which is independent, the following depend on all that precede. These methods, in their general aspect, do not differ from the old developments in powers of the disturbing force, except the operations are so adjusted that the time never escapes from the trigonometric functions. This is accomplished by greatly augmenting the number of the elementary arguments, and by supposing that the rate of motion of each of these is developable according to integral powers of the before-mentioned parameter, or, in some cases, of its square root.

When there is more than one elementary argument, the series obtained in all these ways are pronounced to be generally divergent in the rigorous sense of the word. M. Poincaré brings forward several methods of proof of this. The first depends on the presence of small divisors in the expressions of the coefficients. However, when we do not insist on developments in powers of a parameter, this method of proof has no application. Another method is derived from the principle that two characteristic exponents vanish for every uniform integral that exists. But the integrals which necessitate this conclusion must not only be uniform, they must be valid for every possible case of the problem. Now the integrals known as those of the conservation of living forces and of areas are of this nature; but the integrals derivable from the series of Delaunay, Newcomb, and Lindstedt are valid only for a limited range in the values of the linear variables. For instance, in the problem of the three bodies, if the deformation of the triangle formed by these bodies is such that we cannot find any two sides, one of which sustains to the other an invariable relation of greater to less, we cannot apply the mentioned series. And here it is well to note that the defect of convergence does not arise from the application of the processes 
of integration, but already exists in the development of the perturbative function before integration commences. Thus Delaunay's development of this function at the beginning of his lunar theory is divergent and illusory, unless we have the lunar radius in apogee always less than the solar radius in perigee, and that without regard to the mode of expressing the coefficients. Some of the particular integrals, relied upon by M. Poincaré to establish the vanishing of all the characteristic exponents in case we accept M. Lindstedt's series as valid, lie, so to speak, on the boundary of the domain in which these series are convergent.

In the third place an appeal is made to the alleged nonexistence of analytic and uniform integrals beyond those already known. Were this non-existence clearly established it would decide the question on the side where M. Poincaré has placed himself. But, at least as far as the non-existence of integrals of this nature in a limited domain for the linear variables is concerned, the proof given for it is quite defective. This proof consists in ascertaining how these integrals, supposing them to exist, would behave, should we attempt to derive periodic solutions from them. It is difficult to present this matter without the assistance of algebraic formulæ; nevertheless, it may be attempted. Let there be a number of equations whose left members are formed by the product of two factors. When we pass to a periodic solution, one of these factors becomes zero. What conclusion can we draw from each of the thus modified equations? Evidently one of two things : either the remaining factor of the left member is infinite and the right member indeterminate, or it is finite and the right member a vanishing quantity. Now in case we are obliged to accept the first conclusion, were it only but once, M. Poincaré has demonstrated the non-existence of integrals; but, granting that it is proper in every case to accept the latter conclusion, the demonstration fails. Now he declines to consider the latter alternative, saying that he does not believe that any problem of dynamics, presenting itself naturally, occurs where the right members of the mentioned equations would all vanish. But it should be borne in mind that, while they do not vanish in the general equations, the adjustment of the values of the linear parameters required by the passage to a periodic solution may bring about their vanishing. Thus, in the lunar theory, a periodic solution is brought about by making $e=0, e^{\prime}=0$, and $\gamma=0$, the result is the vanishing of every coefficient having any of these quantities as a factor.

M. Poincaré appeals in another place to the fact that the Lindstedt series, if convergent, would establish the non-existence of asymptotic solutions. But this observation is irrelevant for the reason that the domains of the two things are quite distinct. 
In any case where Lindstedt series are applicable there are no asymptotic solutions, and, where there are asymptotic solutions, Lindstedt's series would be illusory.

We owe much to M. Poincaré for having commenced the attack on this class of questions. But the mist which overhangs them is not altogether dispelled; there is room for further investigation.

\section{KRONECKER'S LINEAR RELATION AMONG MINORS OF A SYMMETRIC DETERMINANT.}

BY PROFESSOR HENRY s. WHITE.

Among the minors of any determinant there exist wellknown identical relations; those of lowest order, the quadratic relations, being readily obtained by the expansion of a determinant in which at least one pair of rows or of columns are identical. If, however, the original determinant is symmetrical, there are identities of a lower order than the quadratic, the linear identities first formally noticed by Kronecker in 1882.* These linear relations, published with no hint as to the manner of their discovery, are suggestive of a certain formula in such constant use as to have become a commonplace in the transformations of the Theory of Invariants of linear substitutions. The latter formula, however, relates to products of two determinant-factors, while Kronecker's is linear; but the latter uses double indices for the constituents, and herein lies the resemblance. By virtue of the ordinary process of multiplication of two determinants, Kronecker's theorem is easily proved to be a consequence from the other identity. Both are equally general, hence it seems likely that the earlier may have been the source of the later. This theory I will develop inductively, using for the sake of brevity determinants of three rows, and obtaining a typical linear relation among three-rowed minors of a six-rowed symmetric determinant.

Form an array of three rows of six constituents each:

$\begin{array}{cccccc}a_{1} & a_{2} & a_{3} & a_{4} & a_{5} & a_{6} \\ b_{1} & b_{2} & b_{3} & b_{4} & b_{5} & b_{6} \\ c_{1} & c_{2} & c_{3} & c_{4} & c_{5} & c_{6}\end{array}$

* Sitzungsberichte der Berliner Akademie, 1882, p. 824. See also C. Runge : Die linearen Relationen zwischen den verschiedenen Subdeterminanten symmetrischer Systeme. Jour. für r. u. a. Math., vol. 93 (1882), pp. 319-327. 